\title{
Hybridization of Off-Axis and In-line High-Resolution Electron Holography
}

Cigdem Ozsoy-Keskinbora ${ }^{1}$, Chris B. Boothroyd ${ }^{2}$, Rafal E. Dunin-Borkowski ${ }^{2}$, Peter A. van Aken ${ }^{1}$ and Christoph T. Koch ${ }^{3}$

${ }^{1}$ Stuttgart Center for Electron Microscopy (StEM), MPI for Intelligent Systems, Stuttgart, Germany.

${ }^{2}$ Ernst Ruska-Centre for Microscopy and Spectroscopy with Electrons and Peter Grünberg Institute, Forschungszentrum Jülich, Jülich, Germany.

${ }^{3}$ Institute for Experimental Physics, Ulm University, Ulm, Germany.

Reconstruction of the complex-valued wave function that gives rise to the probability density of fast electrons in transmission electron microscopy (TEM) seems, at first glance, to be a solved problem. However, it is still challenging when considered more closely. In conventional TEM experiments, only the intensity (i.e., the square of the amplitude) of the wave function can be measured. Direct information about the phase of the wave, which carries information about electrostatic and magnetic fields that the electron has passed through, is lost during detection. In order to recover the phase information, it is necessary to interfere the electron wave function with a reference wave, in order to create an interference pattern. Denis Gabor introduced an approach that could be used to solve this problem 66 years ago [1]. In Gabor's original setup, which is the pioneering scheme for in-line holography, the wave that has been scattered by the specimen (the object wave) interferes with a reference wave propagated along the same axis. Using laser light, Leith and Upatnieks [2] showed that separation of the axes of propagation of the reference and object waves could be used to solve the twin-image problem. Möllenstedt later translated this idea back to electron microscopy, creating the field of off-axis electron holography $[3,4]$.

In-line electron holography, or focal series reconstruction, is now a common method in high-resolution TEM. Although it is very efficient for recovering high spatial frequecny variations in phase, it is inefficient for recovering phase information at low spatial frequencies. In contrast, high-resolution studies are very challenging for off-axis electron holography because the interference fringes must be at least twice as fine as the finest feature of interest in the object to be resolved in the reconstructed wave function. Decreasing the fringe spacing typically increases the noise level for a fixed source brightness, illumination ellipticity and exposure time.

Here, we present a new approach that combines off-axis and in-line holography and allows reliable phase information to be recovered for all spatial frequencies up to a resolution that is approximately twice as high as that obtained in a traditional off-axis electron holography experiment. For a desired signal-to-noise ratio, the required total exposure time is lower than that for traditional high-resolution off-axis electron holography.

Figure 1 shows preliminary results obtained using an FEI Titan TEM operated at $300 \mathrm{kV}$. All holographic data was acquired using round illumination. For off-axis electron holography, the biprism voltage was set to $97.4 \mathrm{~V}$.

Figures 1a, b and c show phase images of a gold particle obtained using in-line electron holography, offaxis electron holography and a combination of the two signals, respectively, for a total exposure time of 16 s. In Figure 1d, power spectrum analysis reveals the increased resolution of the hybrid approach. The 
power spectrum is consistent with off-axis electron holography (Fig. 1b) for low spatial frequencies and with in-line electron holography (Fig. 1a) for high spatial frequencies. Although the noise level in the vacuum region is slightly higher than for in-line electron holography $(0.055 \pi$ vs. $0.046 \pi)$, the recovery of low spatial frequencies is far better than for in-line holography alone. Such noise levels are difficult to achieve using off-axis holography for the exposure time utilized here.

\section{References:}

[1] D. Gabor, Nature vol. 161 (1948), p. 777-778.

[2] E.N. Leith, J. Upatnieks, J. Opt. Soc. Am. vol 52 (1962), p. 1123-1130.

[3] G. Möllenstedt and H. Düker, Naturwissenschaften vol. 42 (1955), p. 41-41.

[4] G. Möllenstedt and H. Wahl, Naturwissenschaften vol. 55 (1968), p. 340-341.

[5] The authors gratefully thank John Bonevich for offering free public use of HolograFREE off-axis holography reconstruction software. The authors gratefully thank Wilfried Sigle, Luis M. Liz-Marzan and Cristina Fernandez-Lopez for samples. CTK thanks the Carl Zeiss foundation and the German Research Foundation (DFG, grant nr. KO 2911/7-1) for financial support. The research leading to these results received funding from the European Union Seventh Framework Programme [FP7/2007-2013] under grant agreement no312483 (ESTEEM2).

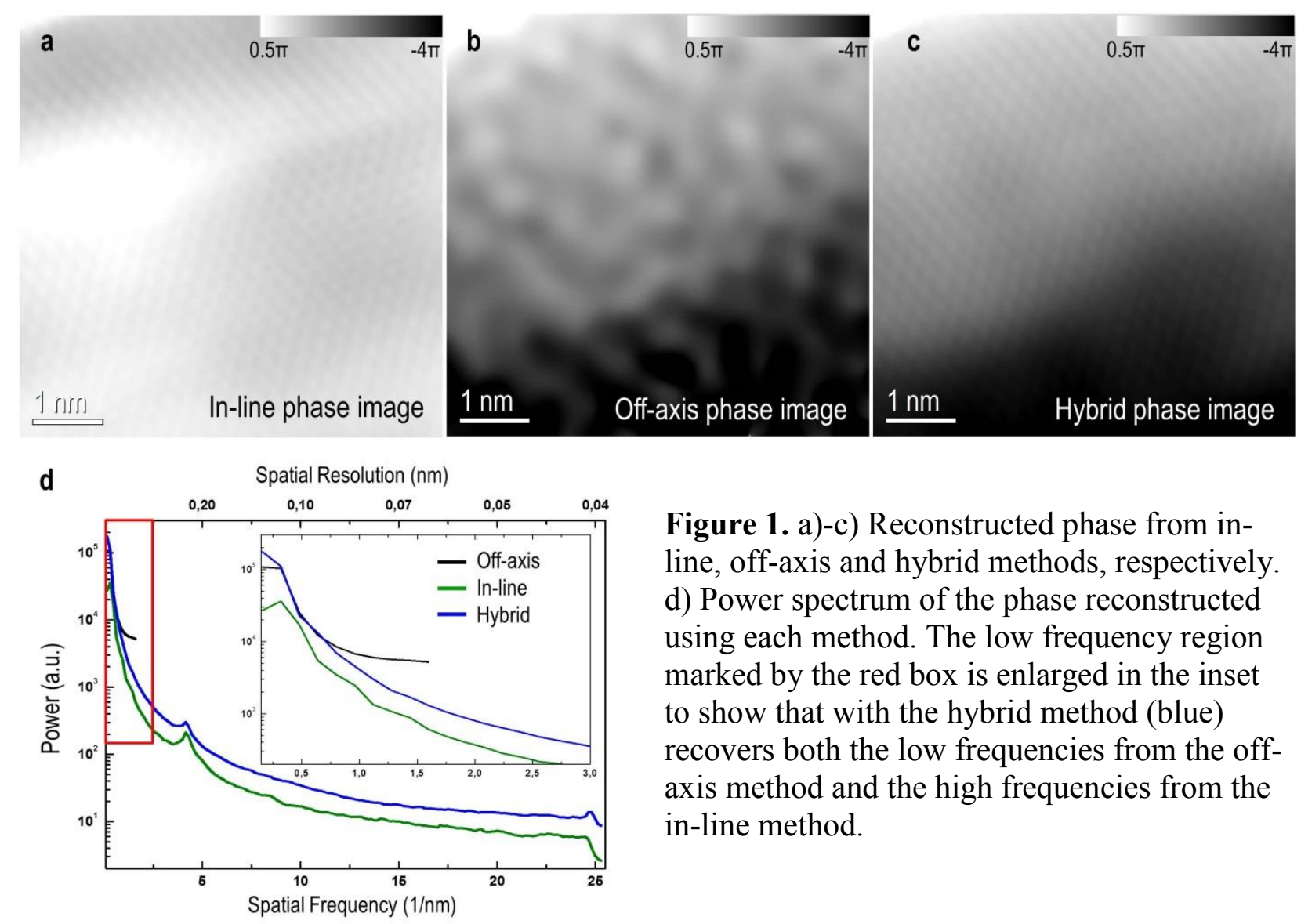

